\title{
Regulatory T cells and the microenvironment of the malignant $B$ cell of chronic lymphocytic leukemia
}

\author{
Georgiana Ene', Ana Maria Vlădăreanu ${ }^{1,2}$, Horia Bumbea ${ }^{1,2}$, Ion Dumitru ${ }^{1}$ \\ ${ }^{1}$ Department of Hematology, Emergency Universitary Hospital, Bucharest, Romania \\ 2"Carol Davila" University of Medicine and Pharmacy, Bucharest, Romania
}

\begin{abstract}
In recent years understanding and modulating the tumor microenvironment (MT) has been the focus of a scientifically and clinically intense study. The role of T regulatory cells (Tregs) were investigated in terms of the suppression of tumor-specific immune responses and the establishment of an immunosuppressive tumor microenvironment (1). Regulatory T cells have a fundamental function in maintaining immune homeostasis in healthy individuals, and in cancer and in particular in haematological malignancies they seem to play a rather controversial role. Furthermore an increased frequency of Treg cells has been associated with tumor progression and has been correlated with an increased risk of death and reduced survival (2). The role of T cells in the pathogenesis of chronic lymphocytic leukemia has recently gained special attention due to the constant interaction between neoplastic B cells with the micromedium substrate and $T$ cells. There is often a relatively large number of regulatory T cells in lymphoid tissues of CLL patients, that could affect the normal immune function (3).
\end{abstract}

Keywords: chronic lymphocytic leukemia, microenvironment, regulatory T cells

\section{INTRODUCTION}

Chronic lymphocytic leukemia (CLL) is one of the most common blood disorder found in adults in the West, with an estimated incidence of about 4.5 new cases per 100,000 people per year (4), the median age at diagnosis is currently considered 72 years, approximately $10 \%$ of patients with CLL have less than 55 years (5).

The disease presents a heterogeneous clinical development. Several factors play an important role in CLL etiology, such as genetic predisposition related to family history, environmental factors and antigens or autoantigens that promote the division of precursor cells and clonal evolution.

There is a genetic predisposition and a family tendency to develop CLL. However, the gene / genes that might support this predisposition and underlie primary transformation events are not yet known, while cytogenetic studies accurately map a number of secondary genetic abnormalities impacting CLL $(6,7)$.
Although the original genetic events are primarily responsible for the first stage of neoplastic transformation, the development and progression of CLL clone is considered to be affected by various signals from the microenvironment that regulate and promote malignant $\mathrm{B}$ cell proliferation and survival. During these processes, other abnormalities may occur after the initial genetic damage, and these new abnormalities may include, for example, somatic mutations of the TP53 or the latest NOTCH1, SF3B1, MyD88 and / or other damage not identified, which allow a more efficient signaling through BCR or other pathways, increased sensitivity to cytokines or chemokines or more efficient traffic at anatomical sites, transforming CLL cells into cells with a more aggressive phenotype (8-13).

Regarding CLL prognosis, the staging clinical systems developed by Rai and Binet are still in use. Additional indicators are available to predict CLL prognosis, that included serum markers (microglobulin $\beta 2$, thymidine kinase, soluble CD23), cell 
markers (CD38, ZAP70) and genetic parameters including cytogenetic abnormalities and mutant status of IGHV genes. The coding of IGHV genes together with BCR and other microtumoral receptors, such as CD38 or CD49d type II transmembrane glycoprotein, as well as the ZAP-70 intracytoplasmic signaling molecule, all negative prognostic factors recognized in CLL, which contribute to an unfavorable prognosis among patients with CLL, who develop a type of aggressive disease requiring immediate therapy, these factors can act in the so-called pseudo-follicular proliferation centers of peripheral lymphoid organs and bone marrow where they play a role in supporting the survival and / or expansion of the leukemic clone $(11,14)$.

\section{CHRONIC LYMPHOCYTIC LEUKEMIA MICROENVIRONMENT}

Chronic lymphocytic leukemia is a prototype of malignant disease that not only depends on intrinsic genetic defects but is also supported by the interactions with spectral cells from micromedium niches (15). CLL can point out three problems:

1. if microenvironment favors the development of the disease or its progression, or is involved in both events (7).

2. identifying and defining microenvironmental elements that influence the malignant clone and relevant molecular pathways (7).

3. clarifying how the micromedium affects cell proliferation relationships and their prolonged survival (7).

Also, CLL is associated with defective T-cell function, leading to the failure of anti-tumor immunity and to increase the susceptibility to infection (16). Typically, CLL is associated with progressive immune deficiency, resulting in hypogammaglobulinemia and deterioration of cellular responses (3,17-18).

Malignant B cells have an aberrant behavior compared to normal B cells in terms of prolifera- tion and apoptosis resistance, having a number of specific characteristics that are described in Table 1. Malignant B cells occur during the various stages of B cell maturation, and some of these cells come from B cells derived from germinal center. During the various stages of development of B cells, T cells are able to control the fate of cell B as follows: can destroy it, can promote cell survival B by regulating anti-apoptotic mechanisms or can induce its proliferation. The tumor malignant $\mathrm{B}$ cells in CLL patients die quickly in vitro, which highlights the importance of the tumor microenvironment in tumor survival (19). Many mechanisms are involved in CLL pathogenesis. For example, Kikushige et al. have proven that hematopoietic stem cells that are self-renewing (HSCs), are involved in CLL pathogenesis and that these HSCs from which CLL cells are derived, generate B cell clones that frequently express surface antigens CD5 and CD23 (21).

Anergy is another important mechanism in maintaining tolerance and has been detected in CLL. Anergy is represented by inactive cells that do not respond to antigens $(22,23)$. Anergy B cells have low levels of surface immunoglobulins and do not respond to conventional stimuli that promote the cellular cycle progression of normal B cells $(7,24)$.

Bone marrow stromal cells, nurse-like cells derived from monocytes (NLC) and the T-cells are key components of the CLL microenvironment. It has been shown that the survival of CLL cells in vitro can be supported by the auxiliary stromal cells $(19,25)$. Tsukada et al. have described in a subset of patients with CLL, blood mononuclear cells which can differentiate in vitro into large, round, adherent cells, which are attached to the leukemia cells and protects them from apoptosis in vitro (26). These cells are known as nurse-like cells (NLCs) and have been shown to be derived from CD14 positive hematopoietic cells, indicating myelomonocytic lineage. NLCs provide a supportive and immune regulatory role in CLL. In addition, they induce the release of CCL3 and CCL4 from CLL cells to at-

TABLE 1. Characteristics of B CLL cells (20)

\begin{tabular}{|l|l|}
\hline Characteristics & Correlations \\
\hline Decrease expression of CD80/CD86 & B cells CLL present least antigen \\
\hline Expression CD200 & Inhibits Th1 cells and induces Treg cells \\
\hline Decrease expression of FASL by FAS & Protect CLL B cells from FAS-mediated cell death; promotes T cell apoptosis \\
\hline Increasing the soluble FAS & Role in CLL progression \\
\hline Secretion of IL-2 and IL-10 soluble receptor & Inhibits Th1 cell differentiation \\
\hline Secretion of IL-6 & $\begin{array}{l}\text { Protects B cells CLL from spontaneous apoptosis;T cells secrete IL-4; consistently positive impact } \\
\text { on the survival of B cell CLL. }\end{array}$ \\
\hline
\end{tabular}


tract other immune cells such as T cells and monocytes $(27,28)$. Also, NCLs induce chemotactism and promote the survival of CLL cells by several incompletely defined mechanisms, such as:

- secretion of chemokine ligand type 12 (CXCL12) and type 13 (CXCL13) (4,29).

- expression of $\mathrm{B}$ cell activation factor (BAFF) $(4,30)$.

- expression of a proliferation promoting ligand (APRIL) $(4,30)$.

- CD31 expression, the CD38 ligand that is expressed in turn by CLL cells (4).

Specialized cells or nurse-like cells of monocytic origin are a component part of the CLL microenvironment that activates $\mathrm{BCR}$ signaling pathways and nuclear factor B (NF-kB) in CLL cells $(4,28)$. Mesenchymal stromal cells (MSCs) of the bone marrow secrete chemokines that regulate the flow of CLL cells, providing further signals that help maintain CLL cell survival and support drug resistance (4). MSCs also play a role in the activity of aggressive markers of the disease such as ZAP70, CD38, CXCR4, and decreased CD20 expression on the surface of CLL cells with implications in the resistance to anti-CD20 antibody treatment $(4,31)$. In addition, MSC can produce more cytokines that can affect hematopoietic cells such as IL-6, IL-7, IL-11, IL-2, IL-14, IL-15, the leukemia inhibitory factor, macrophage stimulation factor, tyrosine kinase 3 ligand (FLT-3), and stem cell factor (3).

Additional cellular micro-environment CLL elements include endothelial cells and follicular dendritic cells (FDC) with role în tissues administration and retention of CLL cells into the tissues. In CLL, FDC inhibits malignant B cell apoptosis via MCL-1 and promotes tumor proliferation (32-33). In vitro, FDCs can promote the survival of CLL cells via CD44 and recruit CLL cells to lymphoid tissues by secreting CXCL13 (3). Stromal cells from the bone marrow summarizes several cytokines (IL-6, IL-7, IL-10, TGF- $\beta$ ), regulates normal B lymphopoiesis, protects CLL B cells from spontaneous apoptosis and apoptosis induced by steroids in vitro, and the interactions between stromal cells and bone marrow microenvironment are important in the progression of the disease or resistance to therapy. VLA-4 and its ligand VCAM-1 are proteins that help the adhesion of B-precursors to stromal cells, and integrins b1 and b2 play a role in the adhesion of CLL B cells to stromal cells (35).

In a normal bone marrow the imbalances that arise between pro- and anti-angiogenic factors result in pathological angiogenesis, which also occurs în CLL pathogenesis. Angiogenic factors are expressed by CLL cells, such as vascular endothelial growth factor (VEGF), which is also an indicator of poor prognosis, VEGF co-receptor neuropilin-1 (NRP1), fibroblast growth factor basic (bFGF) and factor platelet-derived growth (PDGF). The levels of these factors are increased in patients with CLL, and correlate with the disease state and CLL resistance to chemotherapy (4). The explanation is that high levels of angiogenic factors may decrease the stability of the layer of endothelial cells, and thus allowing neo-angiogenesis and the transendothelial migration of CLL cells. Neo-angiogenesis can be targeted in terms of immunomodulatory agents such as lenalidomide therapy, which can reduce both the level of VEGF and bFGF and increase the stability of the endothelium $(4,36)$.

Surface molecules of CLL cells such as the B cell antigen receptor (BCR), chemokine receptors, adhesion molecules, and members of the TNF receptor superfamily (e.g., CD40, BCMA and BAFF-R) are involved in interactions with the tumoral microenvironment ligands, which results in the survival and expansion of the CLL clone and the protection of CLL cells from conventional cytotoxic drugs (37). The cytokines of the TNF family may provide survival signals or may induce apoptosis (38).

\section{Proliferative compartment}

By simply observing that CLL cells progressively accumulate in vivo, but when are grown in vitro undergo apoptosis, questions arise over the microenvironment and its ability to release signals to ensure the survival of malignant cells. This draws attention to the peripheral blood cell compartment where lymphocytes are continually accumulating and which is plausibly to be fed by a upstream proliferation compartment (7). The structure of the proliferative compartment is represented by the focal aggregates of the proliferative prolymphocytes and the proliferative para-immunoblasts, the socalled pseudofolicular or proliferation centers $(7,39)$. Immunohistochemistry studies have revealed that these pseudofolicular centers are CD5 ,$+ \mathrm{Ki} 67+$ cell groups surrounded by new vessels $(7,40)$.

\section{Hematopoietic niche}

Infiltration of CLL cells into bone marrow leads to over-agglomeration and production of factors that distort or affect normal hematopoietic microenvironment. Normal CD34+ haematopoietic stem cells have to compete with CLL cells for 
CXCL12, and a likely consequence is that patients with CLL may have a small number of CD34 + stem cells that may cause granulocytes / macrophages, megakaryocytes and erythrocytes to increase in the bone marrow compared to healthy individuals $(3,41)$. So, the ability to differentiate hematopoietic stem cells seems to be affected by CLL cells. CLL cells produce TNF- $\alpha$, inhibiting the growth of hematopoietic cells in vitro. CLL cells not only infiltrate lymphoid structures but also undermine surrounding cells to become more competent in promoting leukemic cell survival (3). Antigens can activate CLL cells by crosslinking the surface immunoglobulin (sIg) expressed by the leukemic cell. In CLL, sIg expressed by leukemic cells may dictate the fate of the cell and may participate in primary events involved in leukemogenesis $(3,42-43)$.

In vitro, CLL cells secrete various soluble factors that can alter the normal niche of hematopoietic cells. For example, CLL cells express high levels of CXCL8 chemokine and its receptor, unlike normal CD5 + B cells $(3,44)$. Also, CLL cells can express factors that promote cellular survival of CLL, such as CXCL9 and BAFF, and furthermore can express high levels of suppressive immune factors (TGF- $\beta$ and IL-10) and growth stimulating factors (TNF- $\alpha$ and IFN- $\gamma$ ). The secretion of these molecules by CLL cells can configure the micromedium and influence the linkages between CLL cells and other leukemic niche cells (3). Interactions between malignant $\mathrm{B}$ lymphocytes and tumor microenvironment tissues play a major role in the pathogenesis of CLL (Figure 1).

\section{OBJECTIVE}

The objective of this article is to address those essential aspects of Treg cell biology that provide insight into the importance of regulatory $\mathrm{T}$ cells in the development of chronic lymphocytic leukemia and its progress. Another objective is to monitor: the activity of human Treg cells in CLL, their use as progression biomarkers for CLL and their use as therapies in CLL.

\section{DISCUSSIONS}

In many human cancers and in most murine models of tumor growth, the frequency of Treg cells and their suppressor functions are increased compared to those reported for healthy subjects (45). The role of Tregs in tumor growth, metastatic progression, and prognosis of the disease continue to be intensely debated, however there is experimental evidence and clinical argue that Treg cells are involved in the suppression of antitumor immune responses, thereby escaping the tumor from the host immune system $(45,46)$. Thus, the accumulation of regulatory $\mathrm{T}$ cells in situ and in the peripheral circulation of oncological patients can be regarded as an attempt by the tumor to contribute to its own escape from the host immune system by silencing the antitumor effector immune cells (45).

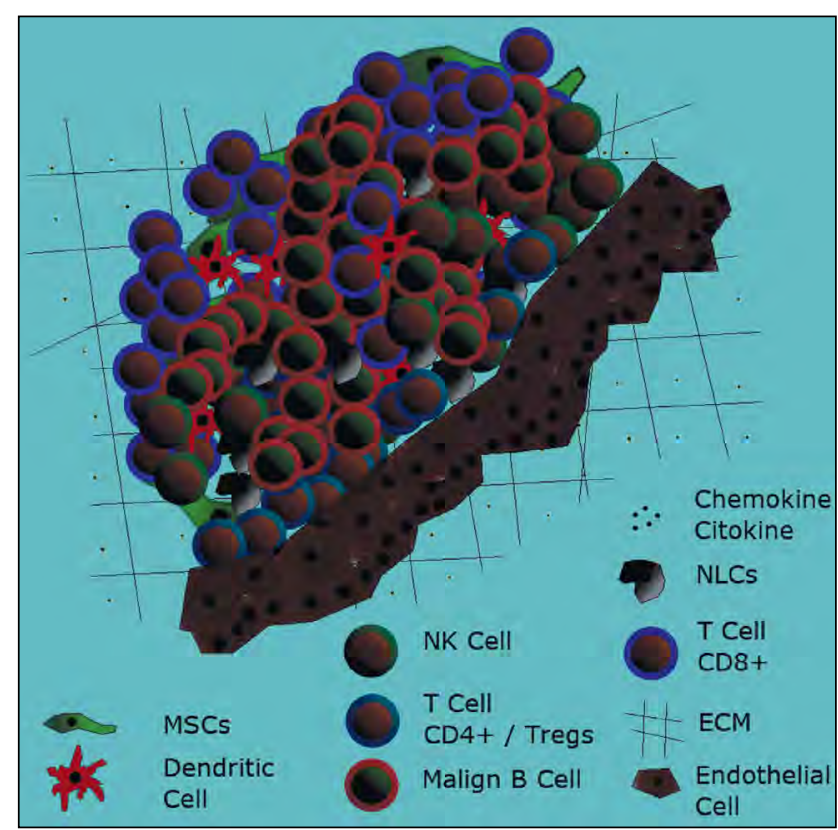

FIGURE 1. Tumoral microenvironment (TM) of malign $B$ cells (79)

Commentary: The neoplastic B cells with normal cells co-evolve to create an immunosuppressive microenvironment that promotes the survival of bone marrow and lymphatic tissues. Tumoral microenvironment is composed of several different cell types adjacent to tumor cells, including stromal cells / mesenchymal stem cells (MSCs), nurse-like cells (NLC), natural killer cells (NK), dendritic cells (DCs) CD4 + and CD8 + $T$ cells and regulatory $T$ cells (Tregs). ECM = extracellular matrix.

Despite the progress made so far in understanding how Treg cells work, many aspects of their interactions with the tumor and other immune or non-immune cells remain obscure. For example, it is not clear whether the Treg cells found in the tumor microenvironment are the same cells that circulate in the periphery or just the functional characteristics are similar to / different from those present in peripheral blood cells (45). Overexpression of several receptors of the Treg cell checkpoints in the tumor microenvironment suggests that these cells acquire significant phenotypic and functional properties once entered into tumor (figure 2). Because of their ability to suppress the functions of antitumor effector $\mathrm{T}$ cells (Teff), Treg cells have been 
perceived as mediators of escape tumor, operating in a silent mode, or are removed if the antitumoral function is to be restored (45,47-48). Regulatory $\mathrm{T}$ cells constitute a small subpopulation of CD4 $+\mathrm{T}$ cells, accounting for about $1-4 \%$ of CD4+ circulating lymphocytes in humans (49).

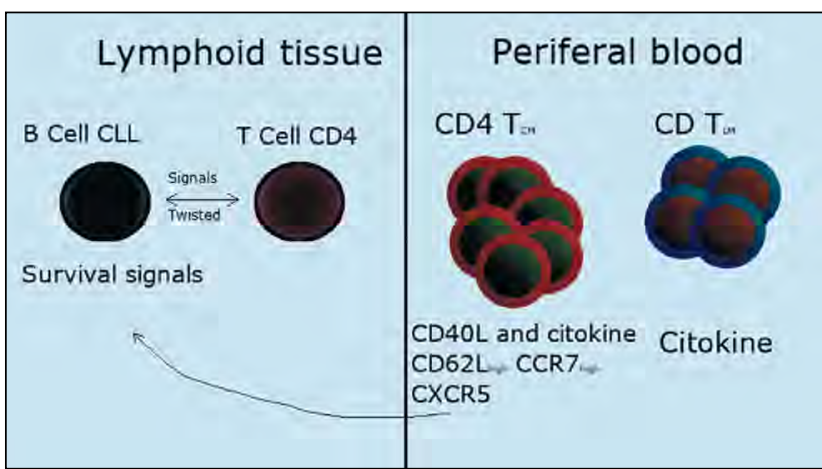

FIGURE 2. Immunological interactions between B CLL cells and CD4 T cells(20)

Initially, Treg cells were defined by CD4 and CD25 expression, but in the last decade for better identification and characterization, it is associated with the expression of other molecules such as CTLA-4 and GITR (2). In general, human Treg cells have been difficult to study for two reasons. First, they represent a minority subset of CD4+ T cells (approximately 5\%) and are not sufficiently numerically available for extensive examinations $(45,50)$. Second, they do not have a specific phenotypic marker to confirm their identity and facilitate their isolation and characterization. Currently, regulatory $\mathrm{T}$ cells can also be characterized by the expression of the transcription factor protein 3 (FoxP3), which is a marker of certainty for Treg cells in mice, but in humans it is not as reliable as it may be absent from certain Treg subsets and present on cells that are not Treg (45,51-52). In addition, FoxP3 is an intracellular protein that is not expressed on the cell surface, so it can not be used to isolate Treg cells $(45,53)$.

To be able to differentiate the two Treg subsets, flowcytometry used expression of surface markers such as CD25hi on the cell surface and intracellular FoxP3, such that pTreg cells exhibit increased heterogeneity regarding the level of expression of the two markers $(45,54)$. Regulation on the surface of receptors $p$ Treg cells inhibitors of checkpoints such as CTLA-4, PD-1, TIM-3, LAG-3, TGF- $\beta$, LAP, GARP or co-expression of CD39 and CD73 is a characteristic that distinguishes pTreg from tTreg $(45,54-56)$. These features of pTreg are particularly highlighted at tumor sites and are interpreted as ev- idence of the ability of these cells to mediate suppression $(45,55)$. And the absence of Treg cell surface markers CD127 or CD26 is often useful for Treg differentiation from Teff CD4+ cells $(45,57-$ 58). Treg also has a phenotype with CTLA-4+, CD62L+, CD127low and GITR+, and other Treg subsets were described by expression of different markers, some of which are associated with increased Treg cell suppression activity, including CD39, HLA-DR and CD103 (59).

Cytokines are necessary for Treg homeostasis, for example IL-2 is one of the key cytokines that thanks to its CD25 receptor helps to proliferate Treg cells, but the role of IL-2 in Treg biology is not clear. Many of the Treg cells express the alpha chain receptor (CD25) of the interleukin 2 receptor (IL-2R), CD25 expression being closely related to IL-2 signaling. Teff cells inside the tumor are those that produce in a great measure IL-2. Regulatory T cells can also produce TGF- $\beta$ which is another key cytokine that plays a role in Teff cell suppression and is also produced by other types of tumor microenvironment cells, immature dendritic cells (DC) and suppressor cells derived from myeloid (MDSCs). Treg cells, having the role of immunosuppressive cells, secrete several suppressive cytokines, such as IL-10, IL-35 and in CLL IL-10 production is higher in the lymph node than in peripheral blood (15).

The role of Treg cells in early body response to cancer cells was mainly investigated in murine studies due to the technical difficulties of studying the biology of Treg before the detection and triggering of human cancer. Oncological experiments targeting murine models offer a variety of knowledge regarding the in vivo interactions of Treg cells, but at the same time raise extra unanswered questions, oriented to the differences between Treg mouse phenotype and Treg human. Human Treg is difficult to phenotype as opposed to Treg murine due to the lack of specific markers and the very wide phenotypic profile of human Tregs (55)., but the combination of markers used for flux phenotyping are: CD3, CD4, CD25, FOXP3, CD127 with CD45RA addition, to clarify Treg activation status, and $\mathrm{Ki}-76$ is useful in vivo and not in vitro as a universal proliferation marker. CD25 and FOXP3 remain the most commonly used markers in the human Tregs phenotype, although not Treg-specific markers.

A minimal phenotypic definition of human Treg cells should include any of the three commonly used immunophenotyping panels:

1. CD25 + CD127loFOXP3 + Treg,

2. FOXP3 + HELIOS + Treg 


\section{FOXP3hi CD45RA- vs. FOXP3int CD45RA+} to distinguish activated Treg cells from nTreg cells $(45,60)$.

A vision that deserves to be taken into consideration is the identification of Treg subsets in cancer patients where differences are observed between the Treg cell phenotype in healthy donors to oncological patients or between the Treg phenotype at the tumor site versus the peripheral blood of the cancer patients (45). Specifically, in oncological patients, the frequency of pTreg cells in the blood and tumor tissues is often increased and Tregs express increased levels of surface markers associated with suppression, for example CD39, CD73, LAP, GARP, COX-2 $(45,61,62)$. Also, these Treg sites have intracytoplasmic expression of perforin molecules, granzyme B and / or IL-10 associated with immune suppression $(45,56)$.

Overall, it is suggested that $p$ Treg cells present in the tumor microenvironment are functionally and phenotypically distinct from tTreg cells. Therefore a definition would be needed to allow for a more precise discrimination of the tTreg from pTreg in oncological patients. Although none of these markers are specific to Treg cells, when combined with surface-bound CD25hi and / or intracytoplasmic FoxP3, they are useful because they allow the evaluation of the potential functions of Treg by flow cytometry (45). Recent efforts to identify a Treg specific marker to distinguish tTreg from pTreg have focused on factor 2 Kruppel (KLF2), a transcription factor that regulates chronic inflammation and is needed in the development of pTreg cells, but not of tTreg cells $(45,63)$.

Although it is known that Treg cells use a variety of mechanisms to mediate suppression, it is not clear whether all Tregs are able to perceive these different mechanisms, or Treg subsets specialized in a certain type of suppression exist. Therefore, the scenario in which different tumors creates microenvironments in which Treg cells are instructed to preferentially adopt the suppression pathway that matches tumor microenvironment schedule is however, plausible (45). The multiple mechanisms by which Treg cells can accumulate in the tumor microenvironment could be:

- Recruitment: tumors recruit Tregs cell due to chemokines secreted by tumor cells and hereditary immune cells; combinations include CCL17 / 22-CCR4, CCL5-CCR5, CCL28CCR10 and CXCL9 / 10/11-CXCR3 (64).

- Extension: in response to factors derived from the tumor microenvironment (TGF- $\beta$,
IL-10), Treg sites can proliferate and expand in situ (64).

- Conversion: Treg production from non-suppressive conventional T cells CD25- under the guidance of TGF- $\beta$ and adenosine (64).

Miyara et al. (64-65) divide regulatory $\mathrm{T}$ cells into three subsets based on their activation and differentiation state:

- effector or activated Tregs cells CD45RAFoxP3hi

- Tregs cells at rest CD45RA+FoxP3lo

- non-suppressive T cells secreting cytokines CD45RA- FoxP3lo or "non-Tregs"

Effector Tregs are a distinct and highly suppressive subset, which modulates the expression of suppressive molecules (GARP / LAP, CD39 / CD73) and immune control points (CTLA-4, TIM-3, GITR, PD-1, LAG-3) (65).

\section{The role of regulatory $\mathrm{T}$ cells in chronic lymphocytic leukemia}

The importance of $\mathrm{T}$ cells in the pathogenesis and development of chronic lymphocytic leukemia is now well established and the role of Treg cells has also been investigated. In addition, a correlation has been described between increased number of Treg cells and clinical and biological characteristics and negative prognosis of CLL. The percentage of Treg cells in CLL is highly variable, and when one takes into account the absolute number, it has been observed that the number of Treg cells in CLL is markedly increased compared with healthy donors (49). Several authors have reported data about Tregs cell in CLL, for example:

- Jak et al. have speculated that the accumulation of Tregs in CLL is due to increased proliferation induced by CD27 / CD70 interaction in lymph node proliferation centers and low apoptosis sensitivity $(49,66)$. Also, using the expression CD4+ CD25 bright CD127 low, they found an increase in the absolute number of Treg cells in CLL and using the Rai classification reported that the percentage of Tregs increased with the stage of the disease $(59,66)$. This group also reported a predominance of Treg CD45RO + cells, suggesting that these cells are derived from memory $\mathrm{T}$ cells in a CD70 dependent manner and accumulate due to diminished apoptosis $(59,66)$.

- Dasgupta et al. tried to establish an optimal threshold for prognostic scores in order to separate the low-risk patients from high risk $(49,67)$.

- D'Arena et al. have found that the absolute number of Tregs cells is an independent predictor of the duration of time until the initiation of the treat- 
ment in the group of Rai stage 0 patients with CLL (49). Their data show that the absolute number of Treg cells is able to identify patients with CLL 0 stage high risk requiring therapy. Their group also tested the influence of green tea (a popular drink in China and Japan) and showed that the number of B lymphocytes and absolute numbers of Treg cells were reduced after oral consumption of green tea extract. Thus, it has been concluded that this product can modulate circulating Treg cells in patients with CLL early-stage and may also delay the progression of the disease (49).

- Rissiek et al. evaluated the composition of circulating $\mathrm{T}$ cell populations and generated $\mathrm{T}$ cell scores showing that the proliferation of suppressor $\mathrm{T}$ cell occurred earlier during monoclonal $\mathrm{B}$ cell lymphocytosis (MBL) $(49,68)$. As the disease progresses from monoclonal lymphocytosis to CLL, $\mathrm{T}$ cell sequential changes occur which gradually compromise $\mathrm{T}$ cell function and contribute to disease progression $(49,69)$. In addition, the absolute number of Treg cells correlates directly with an advanced stage of disease and a greater number of circulating B cells (49).

- Piper et al. on the contrary, they showed that in patients with CLL, Treg cells retain their function and are not influenced by chemotherapy $(49,70)$. A normalization of Treg cell count was observed after fludarabine treatment and in patients treated with lenalidomide, suggesting that drugs are able to modulate cell mediated immunity in CLL (49,71-72).

- Beyer et al. used CD4 + CD25high expression to identify Treg cells and found elevated Treg in CLL compared to healthy donors and observed a correlation between Treg frequency and disease status using the Binet classification $(57,72)$.

- Giannopoulos et al. noted an increase in the percentage of Treg cells in CLL using CD4 + $\mathrm{CD} 25$ high FoxP3 and although they found a correlation between the Treg frequency and the Binet stage, they did not find a significant correlation with ZAP-70 or CD38 (59,73-74).

- Weiss et al. using the CD3 + CD4 + CD25 + CD127 expression to identify Treg cells, found a significant increase in the percentage of Treg in CLL patients compared to healthy control donors. In addition, in their study, the percentage of Treg cells correlates with non-mutant IgVH expression, increased expression of CD38 and specific cytogenetic characteristics. This group also determined that Tregs are an independent predictor of treatment initiation time in these patients $(59,75)$.
CLL cells affect the ability of $\mathrm{T}$ cells to recognize leukemic antigens. In vitro, following direct contact with CLL cells, T-cells from healthy donors lose their ability to form an immunological synapse, i.e., the cell-cell interaction that $\mathrm{T}$ cells produce with antigen-presenting cells (APC) (3). In particular, they lose their ability to polymerize F-actin or recruit accessory cells from places where they are in contact with APC. T cells isolated from CLL patients already show these defects, suggesting that CLL cells similarly affect the ability of T cells to form such immunological synapses in vivo $(3,76)$.

Regarding CLL progression, it appears that effector T cells along with the production of cytokine IL-4 and IFN- $\gamma$ influence it by the fact that it regulates BCL-2 function and protects CLL cells from apoptosis (77-78). A presumption of CLL pathophysiology describes the existence of an intrinsic feedback mechanism in which CLL B cells could induce the generation or accumulation of CD4 T memory cells, which in turn could help them achieve better survival and expansion (20). In the lymph node, neoplastic B cells interact with naive and activated CD4 $\mathrm{T}$ cells, and this action results in the generation or accumulation of CD4 T cells in peripheral blood. With the help of chemokine receptors and CD4 $\mathrm{T}$ memory cell receptors, $\mathrm{T}$ cells can confer on the lymph nodes the survival signals required for B CLL cells (20).

\section{CONCLUSIONS}

Recently published data indicate that the disruption of the function of regulatory $\mathrm{T}$ cells (Tregs) may influence the prognosis of haematological diseases and clinical outcomes. It is considered that Tregs suppress tumor immunity and thus prevent the body's natural ability to control the proliferation of cancer cells. Regulatory $\mathrm{T}$ cells comprise subsets of cells with distinct phenotypic and functional features which helps to escape the tumor from the host immune system. Abnormalities that arise in $\mathrm{T}$ cell subsets may be associated with the progression of chronic lymphocytic leukemia. The attributes of the $\mathrm{T}$ subsets present in the chronic lymphocytic leukemia microenvironment are unclear, they may favor the progression of CLL or may represent the biological basis of CLL specific immunodeficiency. Hence, understanding and discovery of these mechanisms could lead to an improvement in prognostic information and therapeutic approaches in CLL. 
It is also worth noting that the mechanical causes of expansion and change of $\mathrm{T}$ cell direction remain largely unclear, but $\mathrm{T}$ cell defects may be the basis for compensatory expansion seen in patients with CLL. Data from the literature suggests that the interdependence between leukemic B cells, extracellular components of the microenvironment and $\mathrm{T}$ cells modulates the clinical course of disease and

\section{REFERENCES}

1. Deepesh $P$ Lad, Subhash Varma, Neelam Varma et al. Regulatory T-cells in B-cell chronic lymphocytic leukemia: their role in disease progression and autoimmune cytopenias. Leukemia \& Lymphoma, May 2013; 54(5): 1012-1019.

2. Marc Beyer, Joachim L. Schultze Regulatory T Cells: Major Players in the Tumor Microenvironment. Current Pharmaceutical Design, 2009, Vol. 15, No. 16, 1879-1892.

3. Jessie-F Fecteau, Thomas J Kipps. Structure and function of the hematopoietic cancer niche: focus on chronic lymphocytic leukemia. Front Biosci (Schol Ed).(2004); 4: 61-73.

4. Elisa ten Hacken, Jan A Burger. Microenvironment interactions and B-cell receptor signaling in Chronic Lymphocytic Leukemia: Implications for disease pathogenesis and treatment. Biochimica et Biophysica Acta (BBA)- Molecular Cell Research Volume 1863, Issue 3 March 2016, Pages 401-413.

5. B Eichhorst, T Robak, E Montserrat, P Ghia, P Hillmen, M Hallek, C Buske. Chronic lymphocytic leukemia: ESMO Clinical Practice - Guidelines for diagnosis, treatment and follow-up. Annals of Oncology 2015; 26 (Supplement 5): v78-v84.

6. H Dohner, S Stilgenbauer, A Benner, E Leupolt, A Krober, L Bullinger, K Dohner, M Bentz, $P$ Lichter. Genomic aberrations and survival in chronic lymphocytic leukemia N. Engl. J. Med., 343 (2000), pp. 1910-1916.

7. Caligaris-Cappio F. Role of the microenvironment in chronic lymphocytic leukaemia. British Journal of Haematology, 2003, Nov, 123, 380-388.

8. Fabbri G, Rasi S, Rossi D, Trifonov V, Khiabanian H, Ma J, Grunn A, Fangazio M, Capello D, Monti S. Analysis of the chronic lymphocytic leukemia coding genome: role of NOTCH1 mutational activation. The Journal of experimental medicine. 2011;208:1389-1401.

9. Rossi D, Rasi S, Fabbri G, Spina V, Fangazio M, Forconi F, Marasca $\mathrm{R}$, Laurenti L, Bruscaggin A, Cerri M. Mutations of NOTCH1 are an independent predictor of survival in chronic lymphocytic leukemia. Blood. 2012;119:521-529.

10. Rossi D, Bruscaggin A, Spina V, Rasi S, Khiabanian H, Messina M, Fangazio M, Vaisitti T, Monti S, Chiaretti S. Mutations of the SF3B1 splicing factor in chronic lymphocytic leukemia: Association with progression and fludarabine-refractoriness. Blood. 2011;118:69046908.

11. Michele Dal Bo, Riccardo Bomben, Antonella Zucchetto, Giovanni Del Poeta, Gianluca Gaidano, Silvia Deaglio, Dimitar G. Efremov, Valter Gattei. Microenvironmental Interactions in Chronic Lymphocytic Leukemia: Hints for Pathogenesis and Identification of Targets for Rational Therapy. Current Pharmaceutical Design, 2012, 18, 3323-3334.

12. Chiorazzi N, Ferrarini M. Cellular origin(s) of chronic lymphocytic leukemia: cautionary notes and additional considerations and possibilities. Blood 2011; 117: 1781-91.

13. Rossi D, Cerri M, Deambrogi $C$ et al. The prognostic value of TP53 mutations in chronic lymphocytic leukemia is independent of Del17p13: implications for overall survival and chemorefractoriness. Clin Cancer Res 2009; 15: 995-1004.

14. Han T-T, Fan L, Li J-Y, Xu W. Role of chemokines and their receptors in chronic lymphocytic leukemia: Function in microenvironment and targeted therapy. Cancer biology \& therapy. 2014;15:3-9. pathophysiology, mainly by regulating the expansion, survival and differentiation of B CLL cells. It is also important that these interactions can produce qualitative and quantitative changes in the number, function and phenotype of normal $\mathrm{T}$ cells, thus influencing the immune system's ability in patients with CLL.

15. Martijn HA van Attekum, Eric Eldering, Arnon P Kater. Chronic lymphocytic leukemia cells are active participants in microenvironmental cross-talk. Haematologica 2017 Volume 102(9):1469-1476.

16. Riches JC, Davies JK, McClanahan F, Fatah R, Iqbal S, Agrawal S, Ramsay AG, Gribben JG. T cells from CLL patients exhibit features of T-cell exhaustion but retain capacity for cytokine production. Blood 2013 121, 1612-1621.

17. Hamblin AD, Hamblin TJ. The immunodeficiency of chronic lymphocytic leukaemia. Br Med Bull. 2008; 87:49-62.

18. Ravandi F, O'Brien S. Immune defects in patients with chronic lymphocytic leukemia. Cancer Immunol Immunother. 2006; 55(2):197-209.

19. Camilla A Lindqvist, Angelica SI Loskog. T regulatory cells in B-cell malignancy - tumour support or kiss of death. Immunology 2011, 135, 255-260.

20. Rodolfo Patussi Correiaa, Flávia Amoroso Matos e Silva, Nydia Strachman Bacal, Paulo Vidal Campregher, Nelson Hamerschlak, Gustavo P. Amarante-Mendes. Involvement of memory T-cells in the pathophysiology of chronic lymphocytic leukemia. Revista Brasileira de Hematologia e Hemoterapia 2014;36(1):60-64.

21. Kikushige Y, Ishikawa F, Miyamoto T, Shima T, Urata S, Yoshimoto G et al. Self-renewing hematopoietic stem cell is the primary target in pathogenesis of human chronic lymphocytic leukemia. Cancer Cell 2011;20:246e59.

22. R García-Munoz, Jesus Feliu, Luis Llorente. The top ten clues to understand the origin of chronic lymphocytic leukemia (CLL). Journal of Autoimmunity volume 56, January 2015, Pages 81-86, Copyright (C) 2014 Elsevier Ltd. All rights reserved.

23. García-Munoz R, Galiacho VR, Llorente L. Immunological aspects in chronic lymphocytic leukemia (CLL) development. Ann Hematol 2012;91:981-96

24. Caligaris-Cappio F. B-chronic lymphocytic leukemia: A malignancy of anti-self B cells. Blood 1996, 87, 2615-2620.

25. Schulz A, Toedt G, Zenz T, Stilgenbauer S, Lichter P., Seiffert M. Inflammatory cytokines and signaling pathways are associated with survival of primary chronic lymphocytic leukemia cells in vitro: $A$ dominant role of CCL2. Haematologica 2011; 96:408-16.

26. Burger JA, Tsukada N, Burger M, Zvaifler NJ, Dell'Aquila M, Kipps TJ. Blood-derived nurse-like cells protect chronic lymphocytic leukemia B cells from spontaneous apoptosis through stromal cell-derived factor-1. Blood (2000), 96, 2655-2663.

27. A Zucchetto et al. Monocytes/macrophages but not T lymphocytes are the major targets of the CCL3/CCL4 chemokines produced by CD38(+)CD49d(+) chronic lymphocytic leukaemia cell. Br. J. Haematol. 150 (1) (2010) 111-113.

28. JA Burger, MP Quiroga, E Hartmann, A Burkle, WG Wierda, MJ Keating, A Rosenwald. High-level expression of the T-cell chemokines CCL3 and CCL4 by chronic lymphocytic leukemia B cells in nurselike cell cocultures and after BCR stimulation. Blood, 113 (2009), pp. 3050-3058

29. A Burkle, M Niedermeier, A Schmitt-Graff, WG Wierda, MJ Keating, JA Burger. Overexpression of the CXCR5 chemokine receptor, and its ligand, CXCL13 in B-cell chronic lymphocytic leukemia. Blood, 110 (2007), pp. 3316-3325. 
30. M Nishio, T Endo, N Tsukada, J Ohata, S Kitada, JC Reed, NJ Zvaifler, TJ Kipps. Nurselike cells express BAFF and APRIL, which can promote survival of chronic lymphocytic leukemia cells via a paracrine pathway distinct from that of SDF-1alpha. Blood, 106 (2005), pp. 1012-1020.

31. ME Marquez, O. Hernandez-Uzcategui, A. Cornejo, P. Vargas, O. Da Costa. Bone marrow stromal mesenchymal cells induce down regulation of CD20 expression on B-CLL: implications for rituximab resistance in CLL. Br. J. Haematol., 169 (2015), pp. 211-218

32. IM Pedersen et al. Protection of CLL $B$ cells by a follicular dendritic cell line is dependent on induction of Mcl-1. Blood 100 (5) (2002) 1795-1801.

33. K Heinig et al. Access to follicular dendritic cells is a pivotal step în murine chronic lymphocytic leukemia B-cell activation and proliferation. Cancer Discov 4 (12) (2014) 1448-1465.

34. Han T, Barcos M, Emrich L, Ozer H, Gajera R, Gomez GA et al. Bone marrow infiltration patterns and their prognostic significance in chronic lymphocytic leukemia: correlations with clinical, immunologic, phenotypic, and cytogenetic data. J. Clin. Oncol.(1984), 2, 562-570.

35. Irene Munk Pedersen, John C. Reed. Microenvironmental Interactions and Survival of CLL B-cells. Leukemia \& Lymphoma, December 2004 Vol. 45 (12), pp. 2365-2372.

36. R Maffei, S Fiorcari, J Bulgarelli, L Rizzotto, S Martinelli, GM Rigolin, G Debbia, I Castelli, G Bonacorsi, R Santachiara, F Forconi, D Rossi, L Laurenti, GA Palumbo, D Vallisa, A Cuneo, G Gaidano, M Luppi, R Marasca. Endothelium-mediated survival of leukemic cells and angiogenesis-related factors are affected by lenalidomide treatment in chronic lymphocytic leukemia. Exp. Hematol., 42 (2014), pp. 126-136 (e121).

37. Elisa ten Hacken, Jan A. Burger. Molecular Pathways: Targeting the Microenvironment in Chronic Lymphocytic Leukemia - Focus on the B-Cell Receptor. Clinical Cancer Research 20(3) February 1, 2014, 548-56 Published Online First December 9, 2013.

38. Mackay F, Ambrose C. The TNF family members BAFF and APRIL: The growing complexity. Cytokine Growth Factor Rev. (2003), 14, 311-324.

39. Lennert K, Mohri N, Stein H, Kaiserling E, Muller-Hermelink HK. Malignant Lymphomas Other than Hodgkin's Disease. 1978, pp. 119-129. Springer-Verlag, Berlin, Heidelberg, New York.

40. Pileri SA, Ascani S, Sabattini E, Fraternali-Orcioni G, Poggi S, Piccioli M, Piccaluga PP, Gamberi B, Zinzani PL, Leoncini L, Falini B. The pathologist's view point. Part I - indolent lymphomas. Haematologica 2000, 85, 1291-1307.

41. Sala R, Mauro FR, Bellucci R, De Propris MS, Cordone I, Lisci A, Foa $R$, de Fabritiis $P$. Evaluation of marrow and blood haemopoietic progenitors in chronic lymphocytic leukaemia before and after chemotherapy. Eur J Haematol. 1998; 61(1):14-20.

42. Keating MJ, Chiorazzi N, Messmer B, Damle RN, Allen SL, Rai KR, Ferrarini M, Kipps TJ. Biology and treatment of chronic lymphocytic leukemia. Hematology Am Soc Hematol Educ Program. 2003:153-75.

43. Messmer BT, Albesiano E, Efremov DG, Ghiotto F, Allen SL, Kolitz J, Foa R, Damle RN, Fais F, Messmer D, Rai KR, Ferrarini M, Chiorazzi $\mathrm{N}$. Multiple distinct sets of stereotyped antigen receptors indicate a role for antigen in promoting chronic lymphocytic leukemia. $J$ Exp Med. 2004; 200(4):519-25.

44. di Celle PF, Carbone A, Marchis D, Zhou D, Sozzani S, Zupo S, Pini M, Mantovani A, Foa R. Cytokine gene expression in B-cell chronic lymphocytic leukemia: evidence of constitutive interleukin-8 (IL-8) mRNA expression and secretion of biologically active IL-8 protein. Blood. 1994; 84(1):220-8.

45. TL Whiteside, The role of regulatory T cells in cancer immunology. ImmunoTargets and Therapy 2015, vol. 4, pp. 159- 171.

46. Whiteside TL. What are regulatory $T$ cells (Treg) regulating in cancer and why? Semin Cancer Biol. 2012;22:327-334.

47. Whiteside TL, Schuler P, Schilling B. Induced and natural regulatory $T$ cells in human cancer. Expert Opin Biol Ther. 2012;12:1383-1397.

48. Adeegbe DO, Nishikawa $\mathrm{H}$. Natural and induced T regulatory cells in cancer. Front Immunol. 2013; 4:190.
49. Giovanni D’Arena, Candida Vitale, Marta Coscia, Agostino Festa, Nicola Matteo Dario Di Minno, Vincenzo De Feo, Michele Caraglia, Gioacchino Calapai, Luca Laurenti, Pellegrino Musto, Giovanni Di Minno, and Daniela Fenoglio. Regulatory T Cells and Their Prognostic Relevance in Hematologic Malignancies. Hindawi Journal of Immunology Research Volume 2017, Article ID 1832968, 13 pages.

50. Baecher-Allan C, Brown JA, Freeman GJ, Hafler DA. CD4+CD25 high regulatory cells in human peripheral blood. $J$ Immunol. 2001;167: 1245-1253.

51. deLeeuw RJ, Kost SE, Kakal JA, Nelson BH. The prognostic value of FOXP ${ }^{+}$tumor-infiltrating lymphocytes in cancer: A critical review of the literature. Clin Cancer Res. 2012; 18: 3022 -3029.

52. Devaud C, Darcy PK, Kershaw MH. Foxp3 expression in T regulatory cells and other cell lineages. Cancer Immunol Immunother. 2014;63: 869-876

53. Allan SE, Passerini L, Bacchetta R et al. The role of 2FOXP3 isoforms in the generation of human CD4+ Tregs. J Clin Invest. 2005;115: 3276-3284.

54. Schuler PJ, Schilling B, Harasymczuk $M$ et al. Phenotypic and functional characteristics of CD4+ CD39+ FOXP3+ and CD4+ CD39+ FOXP3neg T-cell subsets in cancer patients. Eur J Immunol. 2012;42:1876-1885.

55. Theresa $L$ Whiteside. Induced regulatory $T$ cells in inhibitory microenvironments created by cancer. Expert Opin Biol Ther. 2014 October; 14(10): 1411-1425.

56. Jie HB, Gildener-Leapman N, Li J et al. Intratumoral regulatory T cells upregulate immunosuppressive molecules in head and neck cancer patients. Br J Cancer. 2013; 109:2629- 35.

57. Mandapathil M, Szczepanski M, Harasymczuk M et al. CD26 expression and adenosine deaminase activity in regulatory $\mathrm{T}$ cells (Treg) and CD4(+) T effector cells in patients with head and neck squamous cell carcinoma. Oncoimmunology. 2012;1(5):659-669.

58. Liu W, Putnam AL, Xu-Yu Z et al. CD127 expression inversely correlates with FOXP3 and suppressive function of human CD4+ Treg cells. J Exp Med. 2006;203:1701-1711.

59. Angélique Biancotto, Pradeep K Dagur, John C Fuchs, Adrian Wiestner, C Bruce Bagwell, and J Philip McCoy Jr. Phenotypic complexity of $T$ regulatory subsets in patients with B-chronic lymphocytic leukemia. Mod Pathol. 2012 February; 25(2): 246-259.

60. Santegoets S, Dijkgraaf E, Battaglia A et al. Monitoring regulatory T cells in clinical samples: consensus on an essential marker set and gating strategy for regulatory $T$ cell analysis by flow cytometry. In press 2015.

61. Mandapathil M, Szczepanski MJ, Szajnik M et al. Adenosine and prostaglandin E2 cooperate in the suppression of immune responses mediated by adaptive regulatory T cells. J Biol Chem. 2010;285:27571-27580.

62. Bergmann C, Strauss L, Zeidler R, Lang S, Whiteside TL. Expansion of human $T$ regulatory type 1 cells in the microenvironment of cyclooxygenase 2 overexpressing head and neck squamous cell carcinoma. Cancer Res. 2007;67:8865-8873.

63. Pabbisetty SK, Rabacal W, Maseda D et al. KLF2 is a rate-limiting transcription factor that can be targeted to enhance regulatory T-cell production. Proc Natl Acad Sci U S A. 2014;111(26):9579-9584.

64. Belal Chaudhary, Eyad Elkord. Regulatory T Cells in the Tumor Microenvironment and Cancer Progression: Role and Therapeutic Targeting-Review. Vaccines 2016, 4, 28.

65. Miyara M, Yoshioka Y, Kitoh A, Shima T, Wing K, Niwa,A, Parizot C, Taflin C, Heike T, Valeyre D et al. Functional delineation and differentiation dynamics of human CD4+ T cells expressing the FoxP3 transcription factor. Immunity 2009, 30, 899-911.

66. M Jak, R Mous, EB Remmerswaal et al. Enhanced formation and survival of CD4+ CD25hi FoxP3+ T-cells in chronic lymphocytic leukemia. Leukemia \& Lymphoma, vol. 50, no. 5, pp. 788-801, 2009.

67. A Dasgupta, M Mahapatra, R Saxena. A study for proposal of use of regulatory $T$ cells as a prognostic marker and establishing an optimal threshold level for their expression în chronic lymphocytic leukemia, Leukemia \& Lymphoma, vol. 56, no. 6, pp. 1831-1838, 2015. 
68. A Rissiek, C Schulze, U Bacher et al. Multidimensional scaling analysis identifies pathological and prognostically relevant profiles of circulating T-cells in chronic lymphocytic leukemia. International Journal Cancer, vol. 135, pp. 2370-2379, 2014

69. GD'Arena, G Rossi, MM Minervini et al. Circulating regulatory T cells in "clinical" monoclonal B-cell lymphocytosis. International Journal of Immunopathology and Pharmacology, vol. 24, no. 4, pp. 915-923, 2011

70. KP Piper, M Karanth, A McLarnon et al. Chronic lymphocytic leukaemia cells drive the global CD4+ T cell repertoire towards a regulatory phenotype and leads to the accumulation of $\mathrm{CD} 4+$ forkhead box P3+ T cells. Clinical \& Experimental Immunology, vol. 166, no. 2, pp. 154-163, 2011.

71. BN Lee, H Gao, EN Cohen, X Badoux, WG Wierda, Z Estrov, SH Faderl, MJ Keating, A Ferrajoli, JM. Reuben Treatment with lenalidomide modulates T-cell immunophenotype and cytokine production in patients with chronic lymphocytic leukemia. Cancer, 117 (2011), pp. 3999-4008

72. Beyer M, Kochanek M, Darabi K et al. Reduced frequencies and suppressive function of $\mathrm{CD} 4+\mathrm{CD} 25 \mathrm{hi}$ regulatory $\mathrm{T}$ cells in patients with chronic lymphocytic leukemia after therapy with fludarabine. Blood 2005; 106: 2018- 2025

73. Giannopoulos K, Schmitt M, Wlasiuk P et al. The high frequency of $T$ regulatory cells in patients with $B$-cell chronic lymphocytic leukemia is diminished through treatment with thalidomide. Leukemia. 2008; 22:222-224.
74. Giannopoulos K, Schmitt M, Kowal M et al. Characterization of regulatory $T$ cells in patients with B-cell chronic lymphocytic leukemia. Oncol Rep. 2008; 20:677-682.

75. Weiss EM, Schmidt A, Vobis D et al. Foxp3-mediated suppression of CD95L expression confers resistance to activation-induced cell death in regulatory T cells. J Immunol. 2011; 187: 1684-1691.

76. AG Ramsay, AJ Johnson, AM Lee, G Gorgun, R Le Dieu, W Blum, JC Byrd, JG Gribben. Chronic lymphocytic leukemia T cells show impaired immunological synapse formation that can be reversed with an immunomodulating drug. J. Clin. Invest., 118 (2008), pp. 2427-2437

77. Dancescu M, Rubio-Trujillo M, Biron G, Bron D, Delespesse G, Sarfati $M$. Interleukin 4 protects chronic lymphocytic leukemic $B$ cells from death by apoptosis and upregulates Bcl-2 expression. $J$ Exp Med. 1992;176(5):1319-26.

78. Buschle M, Campana D, Carding SR, Richard C, Hoffbrand AV, Brenner MK. Interferon gamma inhibits apoptotic cell death in $B$ cell chronic lymphocytic leukemia. J Exp Med. 1993; 177(1):213-8.

79. Nicole S Nicholas, Benedetta Apollonio, Alan G Ramsay. Tumor microenvironment (TME)- driven immune suppression in $B$ cell malignancy. Biochimica et Biophysica Acta 1863 (2016) 471-482.

80. Michael Y Choi, Manoj Kumar Kashyap, Deepak Kumar. The chronic lymphocytic leukemia microenvironment: Beyond the B-cell receptor. Best Practice \& Research Clinical Haematology 29 (2016) 40-53 